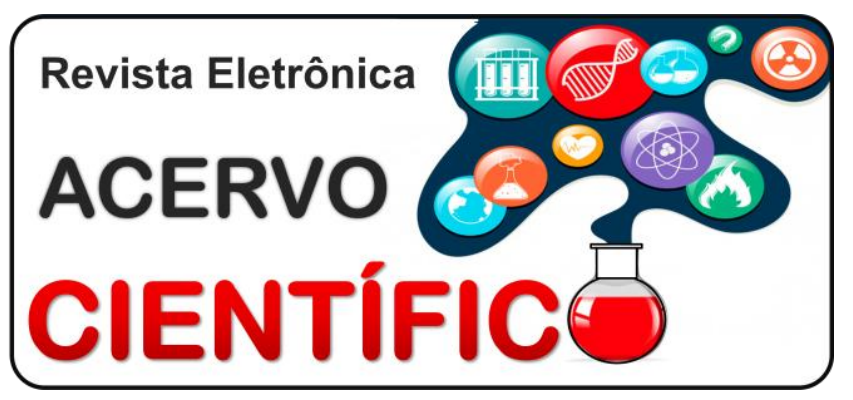

REVISÃO BIBLIOGRÁFICA

Recebido em: 7/2020

Aceito em: 8/2020

Publicado em: 10/2020

\title{
Asma na infância e a importância da integralização do cuidado
}

\author{
Childhood asthma and the importance of comprehensive care
}

Asma infantil y la importancia de la atención integral

\begin{abstract}
Marcela Távora de Freitas ${ }^{1 *}$, Maianna Viana Almeida Aguiar², Anna Clara Fachetti Carvalho ${ }^{3}$, Bianca Fachetti Carvalho ${ }^{4}$, Carolina de Oliveira Bastos ${ }^{1}$, Isabelle Coelho Sampaio ${ }^{1}$, Joyce Cozer de Melo ${ }^{1}$, Letícia Paulo de Souza Cruz ${ }^{1}$, Marina Maciel Rezende ${ }^{3}$, Sara de Oliveira Belmiro'.
\end{abstract}

\begin{abstract}
Resumo: O presente artigo buscou realizar uma revisão acerca do impacto da asma como doença crônica na saúde e na qualidade de vida infantil, bem como esclarecer a importância de cuidados adequados no tratamento da doença. A asma é a doença crônica mais comum observada na infância, envolve fatores genéticos e ambientais e acarreta prejuízos físicos, sofrimento psicológico e social às crianças acometidas. Trata-se de uma inflamação das vias aéreas caracterizada por episódios de obstrução do fluxo aéreo, gerando sintomas como dispneia, sibilância, compressão torácica e tosse. O tratamento apresenta desafios como a falta de informação dos cuidadores e a utilização inadequada dos dispositivos necessários, prejudicando a eficiência no controle da doença. Tal situação aumenta as hospitalizações e interfere na execução das atividades diárias, prejudicando o desenvolvimento escolar e social infantil. Portanto, é necessário um manejo multiprofissional e ações coordenadas em família, serviços de saúde e ambiente escolar, para eficácia no controle da doença e na inclusão desse paciente. Tais medidas buscam promover um tratamento personalizado considerando os aspectos biopsicossociais, o que garante uma melhoria na qualidade de vida da criança asmática.
\end{abstract}

Palavras-chave: Asma, Criança, Qualidade de vida.

\footnotetext{
Abstract: This article sought to review the impact of asthma as a chronic disease on children's health and quality of life, as well as to clarify the importance of adequate care in the treatment of the disease. Asthma is the most common chronic disease in childhood, it involves genetic and environmental factors and causes physical damage, psychological and social suffering to the affected children. It is an inflammation of the airways characterized by episodes of airflow obstruction, generating symptoms such as dyspnea, wheezing, chest compression and cough. The treatment presents challenges such as the lack of information from caregivers and the inappropriate use of devices, impairing the efficiency in controlling the disease. This situation increases with hospitalizations and interferes with the performance of daily activities, impairing children's school and social development. Therefore, multidisciplinary management and coordinated actions in the family, health services and the school environment are necessary to effectively control the disease and

${ }^{1}$ Centro Universitário de Caratinga (UNEC), Caratinga - MG. *E-mail: tavoramarcela@gmail.com

2 União Educacional do Vale do Aço (UNIVAÇO), Ipatinga - MG.

3 Universidade José do Rosário Vellano (UNIFENAS), Alfenas - MG.

4 Universidade de Rio Verde (UniRV), Goianésia - GO.
} 
include this patient. Such measures seek to promote personalized treatment considering the biopsychosocial aspects, which guarantees an improvement in the quality of life of the asthmatic child.

Keywords: Asthma, Child, Quality of life.

Resumen: Este artículo buscó revisar el impacto del asma como enfermedad crónica en la salud y la calidad de vida de los niños, así como aclarar la importancia de una atención adecuada en el tratamiento de la enfermedad. El asma es la enfermedad crónica más común en la infancia, involucra factores genéticos y ambientales y causa daño físico, psicológico y sufrimiento social a los niños afectados. Es una inflamación de las vías respiratorias caracterizada por episodios de obstrucción del flujo aéreo, generando síntomas como disnea, sibilancias, compresión torácica y tos. El tratamiento presenta desafíos como la falta de información de los cuidadores y el uso inadecuado de dispositivos, lo que perjudica la eficiencia en el control de la enfermedad. Esta situación se agrava con las hospitalizaciones e interfiere con el desempeño de las actividades diarias, perjudicando el desarrollo escolar y social de los niños. Por tanto, la gestión multidisciplinar y las acciones coordinadas en la familia, los servicios de salud y el entorno escolar son necesarias para controlar eficazmente la enfermedad e incluir a este paciente. Tales medidas buscan promover un tratamiento personalizado considerando los aspectos biopsicosociales, que garantice una mejora en la calidad de vida del niño asmático.

Palabras clave: Asma, Niño, Calidad de vida.

\section{INTRODUÇÃO}

A asma é uma patologia complexa, a qual está associada a diversos fatores. Trata-se de uma doença obstrutiva crônica recorrente na pediatria, caracterizada pela inflamação das vias aéreas, que evolui para episódios de sibilância, dispneia, compressão no tórax e tosse e, por conseguinte, promove um impacto negativo na qualidade de vida da criança (FONTAN F, et al., 2019; SIQUEIRA KM, et al., 2017).

De acordo com Lozier MJ, et al. (2018), quando não há o controle da asma, o bem-estar dos pacientes é consideravelmente reduzido e aumenta-se o uso de serviços de saúde, como os de urgência e as hospitalizações, o que corrobora para a elevação dos custos do setor. A título de exemplo de como a qualidade de vida é afetada, em crianças de 0 a 17 anos essa enfermidade interfere na execução das atividades diárias e acarreta aproximadamente 10 milhões de dias não frequentados à escola ao ano. É importante ressaltar ainda que no Brasil, no intervalo de 1 ano (de 2014 a 2015), foram registradas cerca de 81.664 hospitalizações decorrentes desta doença em menores de 14 anos (GOMES A, et al., 2017).

Em relação às hospitalizações, conforme Gomes A, et al. (2017), essas podem ser relacionadas à ineficácia do controle da patologia, seja devido à ausência de uma assistência ambulatorial contínua, da interrupção do uso de medicação profilática ou por conta de atitudes preventivas desacertadas no âmbito domiciliar em relação às condições socioeconômica e cultural das famílias. Do mesmo modo, o entendimento insuficiente por parte dos responsáveis a respeito dos cuidados também está relacionado ao aumento da gravidade das manifestações clínicas da asma. Soma-se a isso, a técnica inalatória errônea utilizada pelos pacientes pediátricos ou pelos seus cuidadores também dificulta o controle apropriado dessa doença. Nesse viés, a autoeficácia de cuidadores e púberes asmáticos é necessária para o tratamento, como por exemplo o manejo do uso de espaçadores.

Considerando esse contexto, portanto, por se tratar de uma doença crônica, o tratamento da asma é demorado e requer uma relação satisfatória entre as crianças asmáticas, os responsáveis e a equipe multiprofissional (SIQUEIRA KM, et al., 2017). É imprescindível um tratamento que visa todas as necessidades do paciente pediátrico como o acompanhamento médico, farmacológico, psicológico, fisioterapêutico e o autocuidado por meio do ensino de técnicas para o uso correto de remédios e aparelhos. Ademais, um tratamento integral deve ser ampliado para escola com intuito de promover a inclusão de crianças asmáticas (JENTZSCHA N, et al., 2017). Em concordância com Siqueira KM, et al. 
(2017), é importante pontuar que ações focadas no cuidado centrado no paciente e na família melhoram a comunicação durante as interações clínicas e, consequentemente, acarretam um tratamento mais efetivo.

Perante o exposto, o presente artigo tem como objetivo realizar uma revisão de literatura a respeito do impacto da asma como doença crônica na saúde e na qualidade de vida infantil, bem como esclarecer a importância de cuidados adequados frente ao tratamento da doença.

\section{REVISÃO BIBLIOGRÁFICA}

A asma é a doença crônica mais comum observada no período da infância (SHEIN LS, et al., 2016). Ela se manifesta por episódios de hiperatividade da traqueia e brônquios mediante a presença de estímulos, por consequência ocorre a obstrução do fluxo aéreo. Alterações na geometria e propriedades biomecânicas das vias aéreas são observadas devido a inflamação das mesmas, levando a maior produção de muco, retenção e diminuição do lúmen, ocasionando a persistência da obstrução do fluxo aéreo, que é responsável por causar sintomas como: falta de ar, tosse e episódios recorrentes de sibilância. $\mathrm{O}$ que explica a severidade dos sintomas da asma em crianças é a fisiologia das vias aéreas que, por apresentarem um calibre menor, possuem menos tolerância à hiperatividade característica dessa patologia (FÉLIX AFS, et al., 2019).

Além de evidenciar um impacto social e econômico, a asma é considerada um grave problema de saúde pública, devido à sobrecarga que ocasiona ao sistema. Estima-se que 334 milhões de pessoas no mundo são acometidas com a doença e, no Brasil, mais de $20 \%$ de crianças e jovens já foram diagnosticados, entre a faixa etária de 7 e 14 anos. Assim, a infância é o momento de maior susceptibilidade para o aparecimento da asma, pois no período pré e pós-natal há uma imaturidade da resposta imune inata e adaptativa (COELHO MAQ, et al., 2016).

Segundo Rodrigues APZ e Otto L (2019), o desenvolvimento da asma ocorre por reações alérgicas desencadeadas por uma combinação de fatores genéticos e ambientais. Dessa forma, os pais, familiares e cuidadores devem estar atentos para o reconhecimento dos fatores de risco que podem contribuir para o início das crises asmáticas, principalmente os aeroalérgenos intradomiciliares, tabagismo passivo, medicamentos, obesidade e infecções das vias aéreas superiores (NETO HJC, et al., 2018).

Os ácaros da poeira doméstica, pelos de animais de estimação, baratas, fungos e pólens são os alérgenos intradomiciliares mais frequentes, em que os cuidadores devem adotar medidas preventivas, além de um controle rigoroso da higiene domiciliar. Vale ressaltar que o tabagismo passivo intradomiciliar deve ser banido aos familiares que possuem convívio com os pacientes, visto que a fumaça do cigarro está diretamente relacionada ao desencadeamento das crises ou desenvolvimento da asma (NETO HJC, et al., 2018).

Quanto aos medicamentos, destacam-se a aspirina, anti-inflamatório não esteroidal e $\beta$-bloqueador, que devem ser prescritos de forma individualizada e analisando os riscos e benefícios para cada criança (PIZZICHINI MMM, et al., 2020). A obesidade também se configura como um fator agravante aos pacientes asmáticos, pois o excesso de tecido adiposo ocasiona prejuízos na função pulmonar, gerando impacto no sistema respiratório, interferindo inclusive nos sintomas e tratamento da doença (SILVA AMF, 2019).

As infecções respiratórias virais com rinovírus humano (HRV), especialmente os subtipos $A$ e $C$, são os estímulos mais comuns para a exacerbação da asma. Quando se refere a crianças pequenas e lactentes, a sibilância é frequentemente provocada pelo vírus sincicial respiratório (VSR). Podem estar presentes também, em menor frequência, agentes como o coronavírus e o metapneumovírus humano. Percebe-se que interferons do tipo I são importantes respostas antivirais inatas aos vírus respiratórios e, nesse sentido, em alguns pacientes com asma alérgica, pode-se observar a redução da geração de interferon induzida por vírus a partir de células mononucleares presentes no sangue periférico, células dendríticas plasmocitóides e células epiteliais brônquicas (CASTILLO JR, et al., 2017). Como comprovação, Benedicts FM e Attanasi M (2016), afirmam que tanto em asmáticos atópicos quanto em não atópicos foi encontrada a produção deficiente de interferon por meio das células epiteliais brônquicas em resposta aos rinovírus. 
A asma pode ser classificada em bem controlada, parcialmente controlada e descontrolada. Deve-se avaliar se nas últimas 4 semanas o paciente apresentou a seguinte sintomatologia: presença de sintomas diurnos mais de duas vezes na semana, passou alguma noite acordado devido a asma, fez uso de beta 2 agonista de curta duração (SABA) para alívio dos sintomas mais de duas vezes por semana e limitação das atividades devido a asma. $\mathrm{Na}$ forma bem controlada o paciente não apresenta nenhum dos sintomas mencionados acima, a forma parcialmente controlada é caracterizada pela presença de 1 ou 2 das manifestações citadas e na asma descontrolada o indivíduo relata a presença de 3 ou 4 desses sintomas (GINA, 2020).

Os primeiros passos para confirmação do diagnóstico incluem, além de uma história detalhada, um esboço dos sintomas respiratórios como tosse, chiado e aperto no peito, taquidispneia, tratamentos que já haviam sido instituídos tal como suas respostas, bem como um histórico familiar detalhado que questiona qualquer problema respiratório. Logo após a essa coleta de dados e um exame físico detalhado, é necessário realizar uma espirometria com respostas pré e pós broncodilatadoras (ABUL MH e PHIPATANAKUL $W$, 2019). Torna-se recomendável também iniciar uma investigação sistematizada para observar e minimizar outras comorbidades que possam piorar o controle da doença, tais como refluxo gastroesofágico, obesidade, rinossinusite crônica, polipose nasal, ansiedade e apneia do sono (PIZZICHINI MMM, et al., 2020).

As orientações internacionais sobre a gestão da asma aceitam que o seu tratamento reside no controle atual e no risco de acentuar, sendo estes baseados no controle de sintomas. Neste seguimento, o objetivo do tratamento concentra em minimizá-los, aprimorar a função pulmonar e prevenir as exacerbações. Em crianças, o manejo da asma é analisado com base na manifestação dos sintomas, na restrição das atividades e no recurso à terapêutica de resgate (FÉLIX AFS, et al., 2019).

Nesse contexto, o uso de dispositivos inalatórios faz parte da maioria dos tratamentos e, por isso, a escolha deve ser de acordo com as necessidades de cada paciente. Eles podem ser de vários tipos, como os nebulizadores de jato, os inaladores dosimetrados pressurizados, os inaladores de pó, entre outros (NETO HJC, et al., 2018). Quanto às técnicas dos inaladores de dose calibrada ou de pó seco, são pouco avaliadas durante as visitas pediátricas à asma, porém há evidências que esses instrumentos são utilizados com técnica inadequada e deposição subótima de corticoide inalatório nas vias aéreas, o que acarreta piora do controle da doença (RAMRATNAM SK, et al., 2017). Além disso, o uso de espaçadores é necessário para menores de seis anos, uma vez que evita que partículas se depositem na boca e orofaringe, reduzindo, assim, os efeitos colaterais como candidíase e disfonia (NETO HJC, et al., 2018).

Pacientes pediátricos com asma são um grupo muito desafiador, pois se trata de uma doença crônica que exige uma abordagem multidisciplinar. A terapêutica deve ser encarada como um processo contínuo de monitorização, levando em consideração o paciente como um todo (AHMED H e TURNER S, 2019). Diante disso, é necessária uma atenção integrada, que inclui uma avaliação em pneumologia, alergia, gastroenterologia e otorrinolaringologia, a fim de garantir uma análise completa e individualizada de cada paciente (RAMRATNAM SK, et al., 2017).

Portanto, o tratamento deve ser personalizado incluindo o plano farmacológico, a educação do paciente, métodos de ação e aprendizado do uso de dispositivos inalatórios. Quanto ao plano prescrito pelos especialistas, este deve ser adequado aos padrões socioculturais, idade e gravidade da doença para que as crianças consigam realmente segui-lo (PEREIRA MU, et al., 2015). Assim sendo, é essencial uma boa relação médico-paciente, antes mesmo da escrita do plano terapêutico, de forma a estabelecer conversas interativas e dinâmicas para que a criança se sinta bem orientada e segura (NETO HJC, et al., 2018).

Além disso, o médico deve ser um incentivador da educação do asmático e da família, orientando e reforçando os cuidados e riscos de forma objetiva, visto que, quanto menor o controle da patologia, maior será o comprometimento na qualidade de vida (FONTAN FCS, et al., 2019). Deste modo, precisa estar claro para a equipe de saúde o quanto os pacientes e seus cuidadores sabem sobre a doença, pois a orientação correta é primordial para exercerem suas responsabilidades, buscando assim, um acompanhamento com consultas regulares e automonitorização (NETO HJC, et al., 2018). 
Em relação à dificuldade do controle clínico e funcional, essa enfermidade está estritamente relacionada a uma baixa taxa de adesão ao tratamento por ser considerada uma doença crônica. Foi demonstrado que a escolaridade dos pais é um fator importante, pois, quando não possuem um bom nível de compreensão e educação, dificulta o processo de cuidado (JENTZSCHA N, et al., 2017).

Desse modo, os cuidadores e as crianças asmáticas precisam estar cientes das decisões e dos procedimentos que terão de realizar, o que auxilia no processo de adaptação e na reação emocional dos pacientes quanto às suas restrições. Assim, quanto maior o cuidado e precaução, menor o número de internações, de serviços de emergência, de consultas não programadas e do absenteísmo escolar, colaborando não apenas com o paciente, mas também com o serviço de saúde (GOMES ALA, et al., 2017).

De acordo com Abul MH e Phipatanakul W (2019), a avaliação psicossocial tanto da criança asmática quanto do cuidador é importante. Quadros de ansiedade e dificuldades para lidar com a doença estão muito presentes em crianças com a forma grave da doença, o que acarreta a baixa adesão à medicação e ao seu ineficiente controle. Além disso, a alta morbidade da asma está associada ao estresse psicossocial do cuidador, independente da adesão à medicação. Sintomas depressivos apresentados pelo cuidador podem ocasionar influências no manejo e aceitação dos medicamentos, o que como consequência leva a um controle inadequado da doença.

Já Ahmed H e Turner S (2019), afirmam que a não adesão ao tratamento na sua integralidade inclui-se a negação do diagnóstico, a falta de entendimento sobre a importância de tomar a medicação e seus efeitos adversos, fatores socioeconômicos como o acesso limitado aos profissionais da saúde e à aquisição dos medicamentos, além de técnicas errôneas.

A fim de amenizar as dificuldades do controle, adesão e tratamento da asma, estratégias de orientação são imprescindíveis para um melhor entendimento dos regimes terapêuticos prescritos. Portanto, é necessária uma demonstração prática acerca desses dispositivos tanto para as crianças quanto cuidadores, visto que em um estudo experimental foi observado que $30 \%$ dos pacientes utilizavam os dispositivos inalatórios de forma inadequada. Dentre os erros mais frequentes, destaca-se a distância incorreta do inalador, alteração da velocidade e profundidade da inspiração/expiração, como também a ausência de espaçadores (BRESOLINI DSR, et al., 2020).

Assim, na asma estão inerentes fatores de morbidade como hospitalização prévia, duração e gravidade da doença. Tais fatores se apresentam como a principal causa de absenteísmo no ambiente de ensino, de modo que seu impacto é reconhecido como uma questão de saúde escolar. Existem estudos, ainda, que estabelecem a existência de outros problemas associados, tais como o baixo desempenho acadêmico, problemas de adaptação ao meio escolar e sintomas de stress social e psicológico (FÉLIX AFS, et al., 2019).

Segundo Gomes ALA, et al. (2017), a prática de exercício físico pode funcionar como um fator protetor para as crises asmáticas. Nesse sentido, o esforço físico torna-se um instrumento relevante para o tratamento individualizado, pois avaliará a frequência, intensidade dos sintomas, as limitações e o consumo das medicações de alívio, fornecendo assim, um histórico de controle do estado de saúde da criança que influenciará na sua qualidade de vida (FONTAN FCS, et al., 2019).

Essas práticas permitem que o paciente tenha uma vida mais saudável e com menos restrições na infância, reduzindo riscos futuros e preocupantes dessa patologia crônica, bem como outras comorbidades. Conforme Silva AMF (2019), como o excesso de peso pode intensificar o comprometimento da função pulmonar e prejudicar o tratamento da asma, compreende-se que a atividade física traz benefícios para saúde e inclusão escolar. Nesse sentido, é fundamental que um profissional de saúde oriente os educadores físicos acerca de um treinamento individualizado e personalizado para esses pacientes (NETO HJC, et al., 2018).

A escola é um espaço importante para o aprendizado intelectual e social, onde são construídos laços afetivos que determinam o desenvolvimento psicossocial infantil. No entanto, algumas crianças, como as que possuem asma, precisam de maiores cuidados e, por isso, acabam restritas a certas atividades pelos 
pais, o que pode gerar um sentimento de frustração e exclusão no ambiente escolar. Dentre essas restrições, destacam-se aquelas que intensificam o quadro sintomático da doença, como brincadeiras e atividades físicas que demandam muito da função respiratória (SIQUEIRA KM, et al., 2017).

Além disso, como as crianças passam de 7 a 12 horas por dia nas escolas e creches, acabam sendo, muitas das vezes, expostas a fatores ambientais irritantes que ativam o sistema imunológico e desencadeiam as crises asmáticas (PERMAUL P e PHIPATANAKUL W, 2018; COELHO MAQ, et al., 2016). Essa exposição aos alérgenos pode estar relacionada ao crescente absenteísmo escolar, visto que 49,8\% das crianças faltaram a um ou mais dias de escola nos últimos 12 meses (LOZIER MJ, et al., 2018). Portanto, é fundamental que esse espaço esteja adequado para as necessidades dessas crianças, tendo as salas de aulas sempre ventiladas e com a limpeza adequada, tornando-se um lugar seguro e saudável para todos os tipos de alunos (PERMAUL P e PHIPATANAKUL W, 2018).

A escola deve ser alvo de ações mediadas por uma equipe de saúde que atue na atenção à asma pediátrica. Dessa forma, os educadores precisam ser ensinados a identificar os principais sinais de crise, o manejo adequado da asma e, sobretudo, terem acesso a uma demonstração do uso correto dos inaladores e espaçadores, melhorando assim, a segurança dos pacientes durante a realização das atividades escolares (GOMES ALA, et al., 2017).

Ademais, mesmo com a colaboração escolar, os cuidadores devem tanto comunicar à coordenação sobre essa comorbidade quanto se organizar, para que os procedimentos mais complicados sejam feitos em casa, deixando a escola responsável somente pelo o que é de extrema necessidade. Também seria ideal que os responsáveis deixassem na escola, uma cópia do guia de auto manejo, o plano terapêutico de cada aluno e os dados referentes dos médicos assistentes, uma vez que o controle da asma pode variar e, assim, saberão tomar as devidas providências (NETO HJC, et al., 2018).

Portanto, escola, família e saúde precisam de ações coordenadas a fim de que as repercussões da asma na vida da criança sejam menores ou não tão significantes. Envolver a instituição de ensino é fundamental, pois ela se torna um ambiente de apoio para os pacientes que precisam conviver e se adequar a essa doença crônica (SIQUEIRA KM, et al., 2017).

\section{CONSIDERAÇÕES FINAIS}

Após a análise bibliográfica, conclui-se que a asma é um problema de saúde pública no Brasil, uma vez que se trata de uma doença crônica prevalente na infância e que possui etiologia relacionada tanto a fatores genéticos como ambientais. Sabe-se que é uma patologia que requer o cuidado de uma equipe multiprofissional para diagnóstico, plano terapêutico e educação dos pacientes e familiares, garantindo assim, uma melhor qualidade de vida aos acometidos. Além disso, os estudos demonstram o quanto são importantes ações coordenadas entre família, escola e saúde, visto que o círculo social que envolve a criança asmática precisa estar preparado para qualquer adversidade. Dessa forma, constata-se a necessidade de um apoio maior da atenção primária às crianças e seus cuidadores, a fim de ajudá-los a eliminar erros críticos, que podem agravar a doença.

\section{REFERÊNCIAS}

1. ABUL MH, PHIPATANAKUL W. Severe Asthma in Children: Evaluation and Management. Allergology International 2019; 68(2): 150-157.

2. AHMED H, TURNER S. Severe asthma in children-a review of definitions, epidemiology, and treatment options in 2019. Pediatric Pulmonology, 2019; 64(6): 1-10.

3. BENEDICTIS FM, ATTANASI M. Asma na infância. Eur Respir Ver, 2016, 25: 41-47.

4. BRESOLINI DSR, et al. O uso da visita domiciliar na asma grave pediátrica: estudo experimental randomizado controlado. Revista da Escola de Enfermagem da USP, 2020; 54: 1-7.

5. CASTILLO JR et al. Asthma Exacerbations: Pathogenesis, Prevention, and Treatment. American Academy of Allergy, Asthma \& Immunology. J Allergy Clin Immunol Pract, 2017; 5: 918- 27. 
6. COELHO MAQ, et al. Prevalência e fatores associados à asma em escolares de Montes Claros, MG, Brasil. Ciência \& Saúde Coletiva, 2016; 21(4): 1207-1216.

7. FÉLIX AFS, et al. Controlo da asma infantil: principais fatores associados. Revista electrónica trimestral de Enfermería, 2020; 57: 15-28.

8. FONTAN FCS, et al. Quality of life evaluation and associated factorsin asthmatic children and adolescents attended in a specialized outpatient clinic. Rev Paul Pediatr, 2020; 38:e2018172.

9. GINA. Global Initiative for Asthma. Global Strategy for Asthma Management and Prevention, 2020.

10. GOMES A, et al. Association of self-efficacy of parents/caregivers with childhood asthma control parameters. Rev Esc Enferm USP, 2017; 51: e03282.

11. JENTZSCHA N, et al. Treatment adherence and level of control in moderate persistent asthma in children and adolescents treated with fluticasone and salmeterol. Jornal de Pediatria, 2019; 95(1): 69-75.

12. LOZIER MJ, et al. Assessing health outcomes, quality of life, and healthcare use among school-age children with asthma. Journal of Asthma, 2019; 56(1): 42-49.

13. NETO HJC, et al. Diretrizes da Associação Brasileira de Alergia e Imunologia e Sociedade Brasileira de Pediatria para sibilância e asma no pré-escolar. Arq Asma Alerg Imunol., 2018; 2(2): 163-208.

14. PEREIRA MU, et al. Programa Infantil de Prevenção de Asma: um programa de atenção especializada a crianças com sibilância/asma. J Bras Pneumol, 2016; 42(1):42-47.

15. PERMAUL P, PHIPATANAKUL W. School Environmental Intervention Programs. J Allergy Clin Immunol Pract., 2018; 6(1): 22-29.

16. PIZZICHINI MMM, et al. Recomendações para o manejo da asma da Sociedade Brasileira de Pneumologia e Tisiologia - 2020. J Bras de Pneumol., 2020; 46(1): 1-16.

17. RAMRATNAM SK, et al. Severe Asthma in Children. J Allergy Clin Immunol Pract., 2014; 2(5): 489-500.

18. RODRIGUES APZ, OTTO L. Intervenção da fisioterapia na asma infantil - Revisão de Literatura. Revista de Saúde e Meio Ambiente. Renovare., 2019; 6(2): 9-153.

19. SHEIN SL, et al. Tratamento atual de crianças com asma crítica e quase fatal. Rev Bras Ter Intensiva, 2016; 28(2): 167-178.

20. SIQUEIRA KM, et al. Ser criança com asma: assumindo suas particularidades e lidando com restrições. Rev. Eletr. Enf. [Internet], 2017; 19: 1-10.

21. SILVA AMF, et al. Associação de atividade física com asma em adolescentes brasileiros. Instituto de Estudos em Saúde Coletiva, Universidade Federal do Rio de Janeiro, Rio de Janeiro. 2019. 